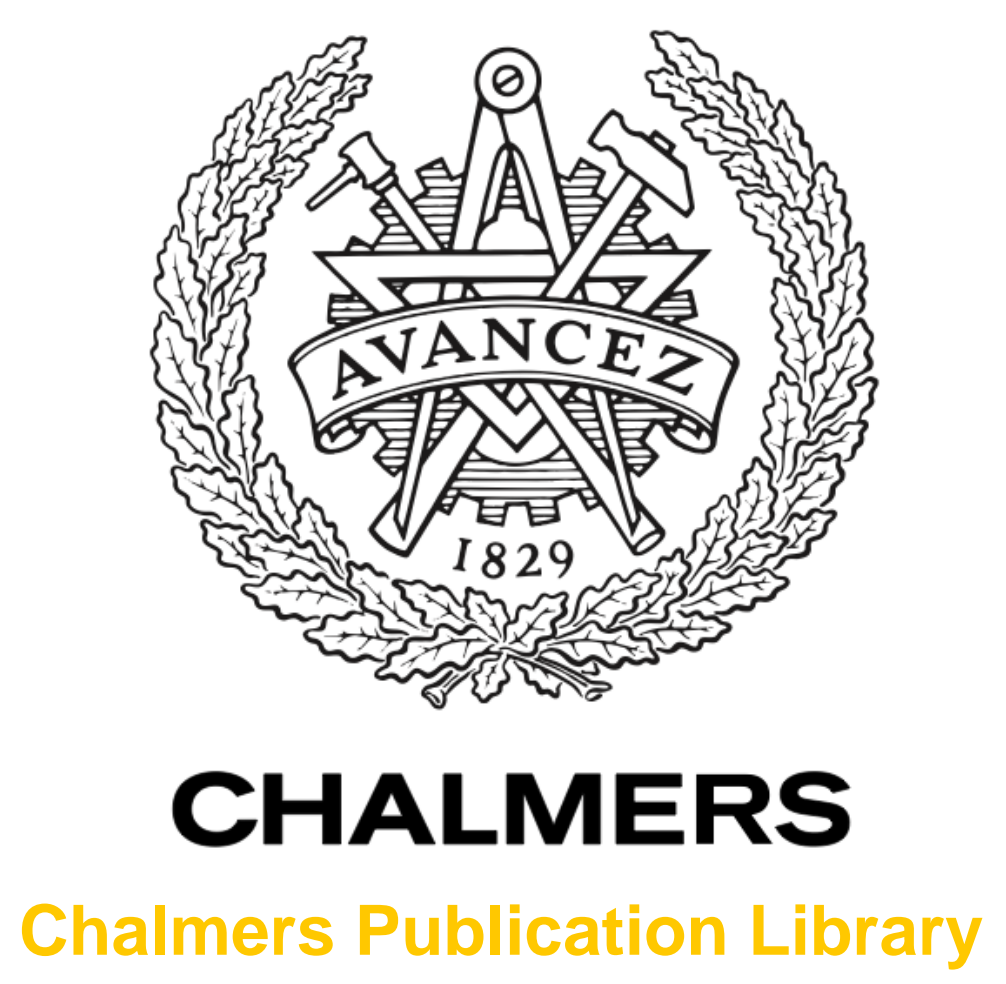

\title{
MIMO systems for turbo coded cooperation over orthogonal and non-orthogonal channels
}

This document has been downloaded from Chalmers Publication Library (CPL). It is the author's version of a work that was accepted for publication in:

11th International Conference on ITS Telecommunications, ITST 2011, St. Petersburg, 23-25

\section{August 2011}

Citation for the published paper:

Farès, H. ; Mohamed, S. ; Langlais, C. (2011) "MIMO systems for turbo coded cooperation over orthogonal and non-orthogonal channels". 11th International Conference on ITS

Telecommunications, ITST 2011, St. Petersburg, 23-25 August 2011 pp. 453-457.

http://dx.doi.org/10.1109/ITST.2011.6060099

Downloaded from: http://publications.lib.chalmers.se/publication/150440

Notice: Changes introduced as a result of publishing processes such as copy-editing and formatting may not be reflected in this document. For a definitive version of this work, please refer to the published source. Please note that access to the published version might require a subscription. 


\title{
MIMO Systems for Turbo Coded Cooperation over Orthogonal and Non-Orthogonal Channels
}

\author{
Haïfa Farès ${ }^{\dagger}$, Sidi Ould Mohamed ${ }^{\dagger}$, Charlotte Langlais ${ }^{\dagger}$, Alexandre Graell i Amat ${ }^{\ddagger}$, Marion Berbineau ${ }^{\S}$ \\ $\dagger$ Department of Electronics, Institut TELECOM-TELECOM Bretagne, Brest, France \\ $\ddagger$ Department of Signals and Systems, Chalmers University of Technology, SE-412 96 Gothenburg, Sweden \\ $\S$ Université Lille Nord de France, IFSTTAR, LEOST, F-59650 Villeneuve d'Ascq, France \\ Email: \{haifa.fares, sidi.ouldmohamed, charlotte.langlais\}@telecom-bretagne.eu, \\ alexandre.graell@chalmers.se, marion.berbineau@ifsttar.fr
}

\begin{abstract}
Multiple-input multiple-output (MIMO) systems can be adopted in cooperative wireless networks to bring further space diversity or/and to improve the throughput of the system. In this paper, we evaluate the performance of MIMO applied to a turbo coded cooperation system. The considered system is studied under two scenarios where the sources transmit all the time over either orthogonal channels or non-orthogonal channels. For the latter, interleave-division multiple-access (IDMA) is used for multi-source detection. The performance improvement are evaluated in terms of reliability (Frame error rate) and efficiency (throughput efficiency), as well as to determine the channel conditions where adopting MIMO in addition to cooperation is beneficial.
\end{abstract}

\section{INTRODUCTION}

The interest in cooperative communications for wireless networks has been rising sharply in the recent years. The major benefit of cooperative communication is that, exploiting the intrinsic broadcast nature of the wireless channel, several nodes help each other by sharing their own antennas in order to transmit information to the destination. This is an efficient way to introduce space diversity. In [1], it was shown that cooperation between sources brings higher data rates as well as higher reliability. A practical cooperation scheme, known as coded cooperation (CCoop), was proposed in [2,3], where channel coding and cooperation were combined. In particular, distributed turbo coding and cyclic redundancy code (CRC) at the partner node was proposed in [3] as an efficient coding scheme for cooperation, called turbo coded cooperation (TCCoop). The partner decodes, interleaves and re-encodes the message prior to forwarding (decode-and-forward protocol). This approach benefits from cooperative diversity, coding and turbo processing gain.

On the other hand, it is known that the achievable capacity of wireless communications increases when multiple transmitter and receiver antennas are used. Therefore, we still witness an increasing interest in the research community on multipleinput multiple-output (MIMO) systems.

This work was performed in the framework of the PREDIT research project MOCAMIMODYN (MOdèles de CAnaux MIMO DYNamiques en tunnels pour des applications transport public). The authors would like to thank the french National Research Agency (ANR) for financial support of this project.

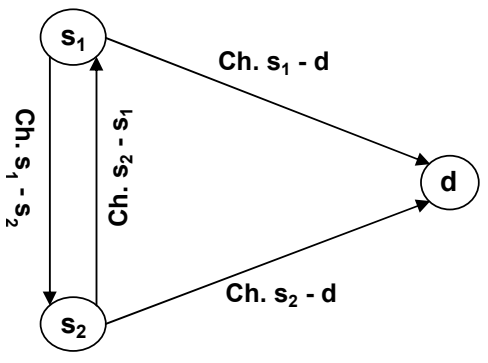

Fig. 1. A three-node cooperative wireless network: Each source acts as a relay for its partner

In this paper, we propose then to study the turbo coded cooperation scheme using MIMO systems in the operating nodes. This study is important in order to check if MIMO techniques still bring further gains besides the distributed space diversity offered by the cooperative system.

Furthermore, compared to the non-cooperative scheme, it is well-known that the coded cooperation may suffer from a throughput degradation, since two phases are always occupied for a single frame. The second phase brings degradation in throughput if the frames from both sources are correctly decoded in the first phase. Therefore, in order to improve the throughput efficiency of such cooperative systems, we consider a more general multi-source cooperative network based on the turbo coded cooperation scheme where both sources transmit to a destination over non-orthogonal multiple-access channel. Several transmitting techniques can be used to deal with this multiple-access interference at the destination. Among them, we consider the use of interleave-division multiple-access (IDMA) for multi-source detection. This choice is dictated by the low complexity of the IDMA receiver. Separation between sources is obtained by the use of a different interleaver for each source. The complexity of the IDMA receiver increases linearly with the number of the sources [4].

The remainder of this paper is organized as follows. The general system model with MIMO systems is defined in Section II. In Section III, we describe the original turbo coded cooperation scheme, over the orthorgonal channels scenario. We further present the more general scheme, where non-orthogonal channels are assumed for the second binding 


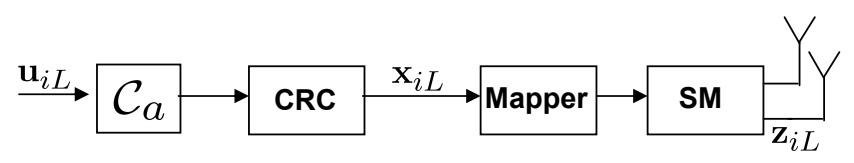

Fig. 2. Local information transmission during the broadcast phase.

cooperation phase. The IDMA technique used for multi-source detection, is explained for this particular context. Finally, numerical results are given in Section $\mathrm{V}$ and some conclusions are drawn in Section VI.

\section{System MOdel}

We consider the wireless relay network depicted in Fig. 1. Two sources $s_{1}$ and $s_{2}$ cooperate to transmit statistically independent data to a single destination $\mathrm{d}$. Both sources operate in the half-duplex setup. Source $\mathrm{s}_{i}(i=1,2)$ can either transmit its own local information (transmission mode) or help the partner node by relaying its information (relaying mode). We denote by $\mathbf{u}_{i L}$ the local data at source $\mathrm{s}_{i}$. Both sources are equipped with two encoders $\mathcal{C}_{a}$ and $\mathcal{C}_{b}$ of rates $R_{a}=1 / 2$ and $R_{b}=1$, respectively. Multiple-antenna may be employed on the operating nodes (sources and destination). In this case, a space multiplexing (SM) operation is performed, generating $\mathbf{z}_{i L}$ and $\mathbf{z}_{i}$, during the broadcast phase and the cooperation phase, respectively. This operation allows to achieve more diversity as well as higher data rates. On the other hand, a maximum likelihood (ML) is used in order to perform MIMO detection.

Thereafter, we describe the considered MIMO channel with $N_{t}$ transmit antennas, $N_{r}$ receive antennas and perfect Channel State Information (CSI) at the receiver but not at the transmitter. All channels are modeled as Rayleigh block fading with additive white Gaussian noise. We denote by $\gamma_{\mathrm{s}_{i} \mathrm{~d}}$ and $\gamma_{\mathrm{s}_{i} \mathrm{~s}_{j}}$ the instantaneous signal-to-noise ratio (SNR) of the $\mathrm{s}_{i}$-to-d channel and of the $\mathrm{s}_{i}$-to- $\mathrm{s}_{j}$ channel, respectively.

The coded cooperation is performed within two transmission phases: a broadcast phase, where both sources transmit their local information, and cooperation phase, where a source may act as a relay helping the partner by forwarding its information. Therefore, to still operate in the half-duplex setup, the sources have to transmit in an orthogonal way for the first transmission phase (broadcast phase). After the broadcast phase, the received observations from $\mathrm{s}_{i}$ at $\mathrm{s}_{j}(i, j=1,2$ with $i \neq j$ ) and at the destination $d$, can be written, respectively as

$$
\begin{gathered}
\mathbf{y}_{\mathrm{s}_{i}}^{\mathrm{s}_{j}}=\mathbf{H}_{\mathrm{S}_{\mathbf{i}} \mathrm{s}_{\mathbf{j}}} \mathbf{z}_{\mathbf{i L}}+\mathbf{n}_{\mathrm{s}_{\mathbf{i}} \mathrm{s}_{\mathbf{j}}} \\
\mathbf{y}_{\mathrm{s}_{i}}^{\mathrm{d}}=\mathbf{H}_{\mathrm{s}_{\mathbf{i}} \mathrm{d}} \mathbf{z}_{\mathbf{i L}}+\mathbf{n}_{\mathrm{s}_{\mathbf{i}} \mathrm{d}}
\end{gathered}
$$

The channel matrices $\mathbf{H}_{\mathrm{S}_{\mathbf{i}} \mathrm{S}_{\mathbf{j}}}$ and $\mathbf{H}_{\mathrm{s}_{\mathbf{i}} \mathrm{d}}$ of dimensions $\left(N_{t}, N_{r}\right)$ are assumed to be constant over the transmission of a source frame, i.e., over all transmission phases. This channel model is considered in order to investigate the ability of the distributed spatial diversity to enhance link level performance in scenarios where temporal diversity is limited or unavailable. These channel matrices turn into scalar coefficients

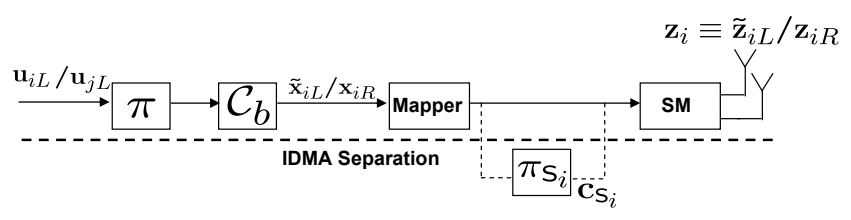

Fig. 3. Relayed information transmission during the cooperation phase.

when single-input single-output (SISO) systems are considered $\left(N_{t}=N_{r}=1\right)$.

The orthogonal and non-orthogonal channels differ only in the cooperation phase, where signals from both sources are completely separated (orthogonal channel) or superposed (non-orthogonal channel).

\section{A. Orthogonal channels}

The sources transmit on orthogonal channels, e.g., time division multiple access (TDMA), which allows the destination to separately detect each source. After the cooperation phase, the received observations from partner nodes at the destination $d$ are disjoint and can be written as

$$
\begin{aligned}
& \mathbf{y}_{\mathrm{s}_{1}}^{\mathrm{d}}=\mathbf{H}_{\mathrm{s}_{1} \mathrm{~d}} \mathbf{z}_{1}+\mathbf{n}_{\mathrm{s}_{1} \mathrm{~d}} \\
& \mathbf{y}_{\mathrm{s}_{2}}^{\mathrm{d}}=\mathbf{H}_{\mathrm{s}_{\mathbf{2}} \mathrm{d}} \mathbf{z}_{2}+\mathbf{n}_{\mathrm{s}_{2} \mathrm{~d}}
\end{aligned}
$$

From equations (3) and (4), we note that $s_{i}$ transmits over its own uplink channel the relayed information $\mathbf{z}_{i}, i=1,2$. According the transmission mode, $\mathbf{z}_{i}$ can convey two different information: $\mathbf{z}_{i R}$, the relayed information of $\mathrm{s}_{j}$ (relaying mode), and $\tilde{\mathbf{z}_{i L}}$, another version of its local information (transmission mode).

\section{B. Non-Orthogonal channels}

Both sources transmit simultaneously during the cooperation phase. The received signal at the destination is the superposition of the transmitted vectors $\mathbf{z}_{i}$ (weighted by the channel coefficients) from the different sources, can be written as

$$
\mathbf{y}_{\mathrm{s}}^{\mathrm{d}}=\mathbf{H}_{\mathrm{s}_{1} \mathrm{~d}} \mathbf{z}_{\mathbf{1}}+\mathbf{H}_{\mathrm{s}_{\mathbf{2}} \mathrm{d}} \mathbf{z}_{\mathbf{2}}+\mathbf{n}_{\mathrm{sd}}
$$

\section{Orthogonal Turbo Coded CoOperation}

In the following, we briefly describe the coded cooperation scheme of Fig. 1. Since we assume TDMA, all channels are orthogonal. Without loss of generality, we focus on the information generated at source $\mathrm{s}_{1}$. The transmission of user data $\mathbf{u}_{i L}$, of length $K$ bits, is performed over two phases. In the first phase, referred to as the broadcast phase, source $\mathrm{s}_{1}$ encodes $\mathbf{u}_{1 L}$ by $\mathcal{C}_{a}$ into codeword $\mathbf{x}_{1 L}$, of length $N_{a}=K / R_{a}=2 K$ bits. $\mathbf{x}_{1 L}$ is augmented with a cyclic redundancy code (CRC) in order to facilitate error detection and transmitted over the wireless channel. For simplicity, in the remainder of the paper, when referring to a codeword $\mathbf{x}$, we shall assume that it includes a CRC. The broadcast phase for source $\mathrm{s}_{i}$ is described in Fig. 2. Thereafter, generated codewords at both sources are Gray-mapped into complex symbols before passing the SM 


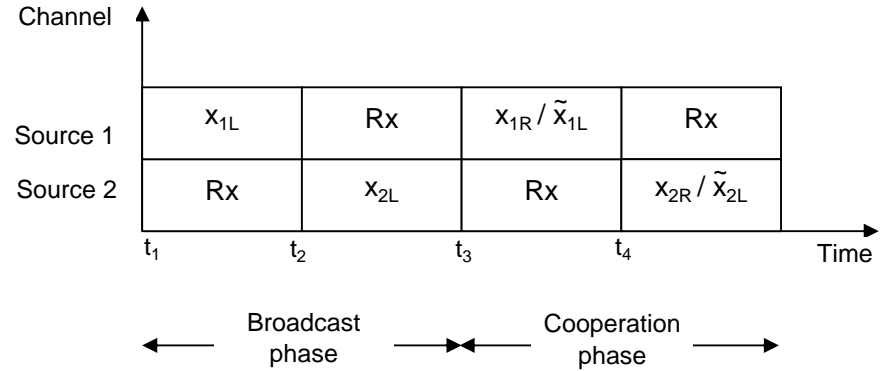

Fig. 4. The transmission time of the coded cooperation scheme is divided into two totally orthogonal phases: broadcast phase and cooperation phase.

block. Due to the broadcast nature of the wireless channel both the destination and the partner node $s_{2}$ receive a noisy observation of $\mathbf{x}_{1 L}$. If decoding is successful at node $s_{2}$ (i.e $\mathrm{s}_{2}$ is able to regenerate $\mathbf{u}_{1 L}$ ), it switches to the relaying mode; at the cooperation phase, $\mathbf{u}_{1 L}$ is then interleaved through an interleaver $\pi$ into $\mathbf{u}_{2 R}=\pi\left(\mathbf{u}_{1 L}\right)$, encoded by $\mathcal{C}_{b}$ into $\mathbf{x}_{2 R}$ and forwarded to the destination after being modulated and restructured into a space block vector. On the other hand, if decoding is not successful, $\mathrm{s}_{2}$ operates in the transmission mode (non-cooperative); at the second phase $\mathbf{u}_{2 L}$ is then interleaved by $\pi$ into $\tilde{\mathbf{u}}_{2 L}$, encoded by encoder $\mathcal{C}_{b}$ into $\tilde{\mathbf{x}}_{2 L}$, modulated and space multiplexed before being forwarded to the destination. A similar operation is performed at node $\mathrm{s}_{1}$ during the second phase.

Notice that with some abuse of language we call the second phase the cooperation phase. However, in this phase source $\mathrm{s}_{i}$ may work either in the transmission mode or in the relaying mode, depending on the CRC check. The cooperation phase for source $s_{i}$ is described in Fig. 3. The figure illustrates both cases: the typical cooperation phase over orthogonal channels and the cooperation phase over non-orthogonal channels, where a specific random interleaver is used to ensure IDMA seperation between superposed sources. Unlike some minor differences, this system is similar to the well known space time-bit interleaved coded modulation (ST-BICM).

With reference to source $s_{1}$ four cases are possible at the destination:

1) decoding at nodes $s_{1}$ and $s_{2}$ is successful: the destination receives two noisy observations of $\mathbf{x}_{1 L}, \mathbf{y}_{1 L}^{\mathrm{d}}$ and $\mathbf{y}_{2 R}^{\mathrm{d}}$, from $\mathrm{s}_{1}$ and $\mathrm{s}_{2}$, respectively. Notice that $\mathbf{x}_{1 L}$ and $\mathbf{x}_{2 R}$ form a codeword of a (distributed) turbo code, where the first component codeword is generated at $s_{1}$ and the second component codeword is generated at $\mathrm{s}_{2}$. Therefore, the relay network of Fig. 1 behaves as a distributed (over space) turbo code, and the destination can estimate $\mathbf{u}_{1 L}$ in an iterative fashion.

2) decoding at node $s_{1}$ is successful, decoding at node $\mathrm{s}_{2}$ fails: the destination attempts to estimate $\mathbf{u}_{1 L}$ by decoding only codeword transmitted during the first phase.

3) decoding at node $s_{1}$ fails, decoding at node $s_{2}$ is successful: the destination receives three noisy observations

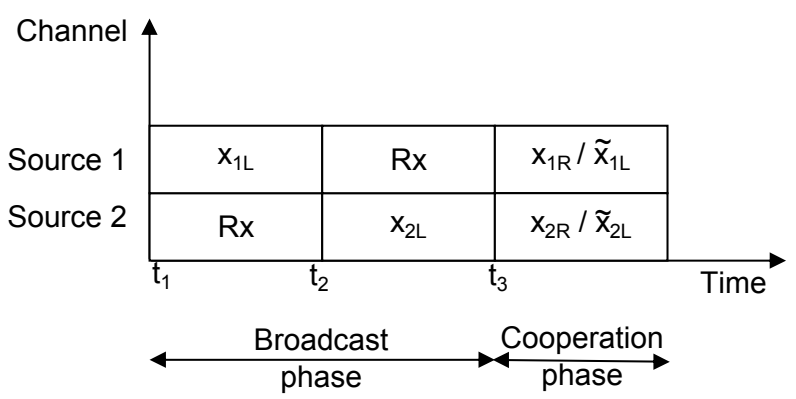

Fig. 5. The transmission time is yet divided into two phases: orthogonal broadcast phase (two time slots) and a non-orthogonal cooperation phase (a unique time slot allocated for both sources).

of $\mathbf{x}_{1 L}$, which are decoded in a turbo fashion.

4) decoding at nodes $s_{1}$ and $s_{2}$ fails: the destination receives two noisy observations of $\mathbf{x}_{1 L}$. Therefore, a distributed (over time) turbo code is obtained and the destination can estimate $\mathbf{u}_{1 L}$ in an iterative fashion.

The four cases are summarized in Fig. 4.

\section{Non-Orthogonal Turbo CODED COOPERATION}

A throughput degradation characterizes typically cooperative communications due to a supplementary fixed cooperation phase. This drawback may be circumvented by reducing the transmission time of this critical phase. The most trivial solution is transmission over non-orthogonal channels. However, this non-orthogonal multi-source transmission via IDMA is limited to the second phase (cooperation phase). This is imposed by the half-duplex setup under which the sources are constrained to operate. The transmission time is illustrated in Fig.5.

The IDMA technique, proposed in [4], can be considered as a special case of the CDMA technique, where the different sources are distinguished by means of interleavers (see Fig. 3). Add to that, IDMA inherits many advantages from CDMA, in particular, diversity against fading and mitigation of the interference problem. Originally, unlike the CDMA technique, a same spreading code is used for all the sources. Besides, it was proved that this spreading code is not needed if a prior low-rate channel code is used. However, in our work, no spreading code is employed while a high-rate is used. This is due to the fact that a part of the information, transmitted during the broadcast phase, is already separated thanks to orthogonal channels. The key principle of IDMA is then the use of interleavers $\pi_{\mathrm{s}_{i}}$ to distinguish between different sources leading to an iterative multi-source separation at the receiver, described below.

\section{A. IDMA receiver structure}

In the receiver, two main blocks are used: an elementary signal estimator (ESE) that exchanges extrinsic information with two a posteriori probability $(A P P)$ decoders, $\mathcal{C}_{\mathrm{S}_{i}}^{-1}, i=$ 1,2. A global iterative process is then applied to recover the information of both sources. Due to the use of interleavers $\pi_{\mathrm{s}_{i}}$, the ESE operation can be carried out by a chip-by-chip detection algorithm [5]. 


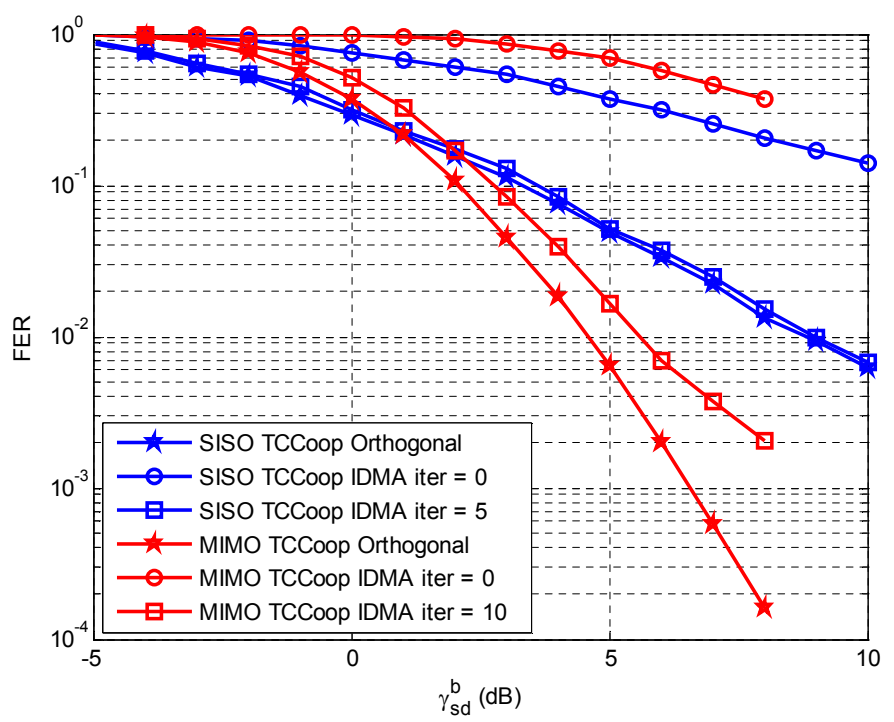

Fig. 6. Frame error rate curves of turbo coded cooperation with SISO and MIMO systems for $\gamma_{\mathrm{ss}}^{b}=\infty \mathrm{dB}$.

The outputs of the ESE and $\mathcal{C}^{-1}$ are extrinsic LLRs about source codeword. The ESE computes the extrinsic information $L_{e \text {,ESE }}$ using the chip-by-chip detection algorithm, described in [4]. Furthermore, an intrinsic iterative process is performed to obtain a distributed turbo decoding.

\section{Performance Evaluation}

In this Section, we give frame error rate (FER) results and throughput performance of MIMO systems applied to turbo coded cooperation scheme and compare them with the turbo coded cooperation where no multi-antenna nodes are assumed, i.e., SISO systems. For the examples here we consider the rate- $1 / 2$ convolutional encoder with generator polynomials $(1,15 / 13)$ in octal form for $\mathcal{C}_{a}$ and the rate- 1 convolutional encoder with generator polynomial $(17 / 13)$ in octal form for $\mathcal{C}_{b}$. The information block length is $K=128$ bits, symmetric $\mathrm{s}_{i}$-to-d channel $\left(\gamma_{\mathrm{s}_{1} \mathrm{~d}}=\gamma_{\mathrm{s}_{2} \mathrm{~d}}\right)$ is assumed and a random interleaver is used for $\pi$. In order to operate at the same spectral efficiency, for SISO setup a quadrature phase shift keying (QPSK) modulation is used, while for MIMO $2 \times 2$ setup $\left(N_{t}=N_{r}=2\right)$, a binary phase shift keying (BPSK) modulation is considered.

In Fig. 6 FER curves are given for perfect inter-source channel quality $\left(\gamma_{\mathrm{ss}}^{b}=\infty\right)$ as a function of $\gamma_{\mathrm{sd}}^{b}$, where $\gamma^{b}=\gamma / R$, being $R$ the rate of the system. This particular inter-source channel is considered in order to highlight MIMO contributions over a cooperative system. Thus in order to perform cooperation continuously, an error-free inter-source channel have to be assumed. Both sources operate all the time under the first cooperative case described in Section III.

Note that the rate of the system depends on the overall code rate $\left(R_{\mathrm{c}}\right)$, modulation order $\left(R_{\mathrm{m}}\right)$ and the considered channel (orthogonal or non-orthogonal). For instance, for an orthogonal TCCoop, the overall rate is fixed to $R=1 / 3$, it corresponds to 6 different time slots allocated to transmit two

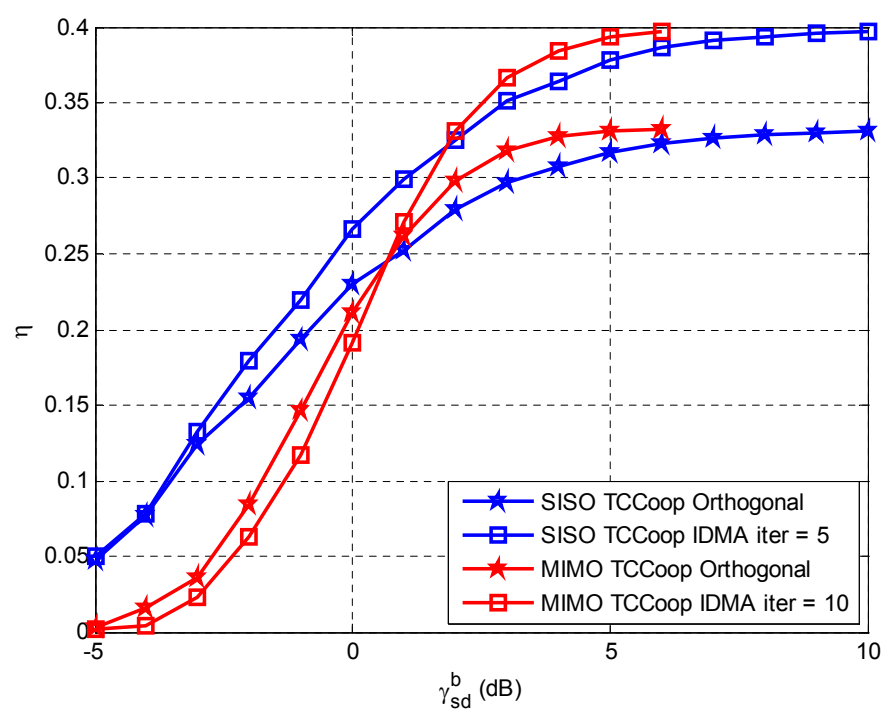

Fig. 7. Throughput efficiency curves of turbo coded cooperation with SISO and MIMO systems for $\gamma_{\mathrm{ss}}^{b}=\infty \mathrm{dB}$.

local frames of two sources. On the other hand, for a nonorthogonal TCCoop, both local frames at cooperating sources take 5 different time slots to reach the destination; then, the overall rate is fixed to $R=2 / 5$.

Adding MIMO spatial multiplexing to the SISO TCCoop scheme yields $4.5 \mathrm{~dB}$ gain at $\mathrm{FER}=10^{-2}$. We note then that cooperative systems still benefit from space diversity offered by multi-antenna network deployment, since the slope of the curve is changed. However, this gain is not exclusively due to space diversity. It is also the result of better system throughput obtained thanks to spatial multiplexing operation. Furthermore, FER performance of SISO TCCoop system is slightly affected when operating over non-orthogonal channels. For instance, a very low degradation persist after 5 iterations of IDMA process aiming on source signal separation (interference cancellation). This performance degradation in terms of FER is larger for MIMO TCCoop system after 10 IDMA iterations. Therefore, more iterations are needed to better separate both sources.

Throughput performance is reported in Fig. 7. In particular, we plot the throughput efficiency $(\eta)$, defined as [6]

$$
\eta=\rho T
$$

where $\rho$ is the reliability, defined as the ratio between the number of accepted bits and the number of received bits, and $T$ is the throughput of the system, defined as the ratio between the average number of accepted bits and the total number of bits sent over the channel per unit time. Therefore, the throughput efficiency is defined as the ratio between the number of information bits correctly transmitted per transmitter and channel time allocation.

The throughput efficiency of the turbo coded cooperation system over orthogonal channels is limited to $\eta \leq 1 / 3$, since two phases are always allocated for the transmission of a packet, hence $R=1 / 3$. A sizable throughput efficiency increase is obtained over non-orthogonal channel based on 
IDMA technique. In this case, the throughput efficiency is limited to $R=2 / 5$ since better transmission delay is obtained when the same time slot is allocated to both sources to perform the cooperation phase. The FER degradation observed over non-orthogonal channel with respect is largely compensated by the throughput gain. Therefore, the throughput efficiency behavior is globally better for the non-orthogonal TCCoop than the orthogonal TCCoop, for both SISO and MIMO setups. This is mainly observed for high-SNR regime. Asymptotically, the throughput efficiency gain of non orthogonal TCCoop systems with respect to the orthogonal one is about $20 \%$. For low-SNR regime, MIMO TCCoop systems behave at the best as SISO TCCoop systems, and often worst. This observation is congruent with results in terms of FER depicted in Fig. 6. For instance, under the orthogonal scenario, MIMO TCCoop system outperforms SISO one for $\gamma_{\mathrm{sd}}^{b}>1 \mathrm{~dB}$. On the other hand, under the non-orthogonal scenario, MIMO TCCoop system still performs worts than SISO TCCoop system for $\gamma_{\mathrm{sd}}^{b} \leq 2 \mathrm{~dB}$.

\section{CONCLUSIONS}

In this paper, we considered turbo coded cooperation with MIMO systems and we compared it with the original turbo coded cooperation where no multi-antenna nodes are deployed.
We studied the considered system over two scenarios: orthogonal (TDMA) and non-orthogonal (IDMA). First, it was shown that turbo coded cooperation still benefits from spatial diversity offered by MIMO systems. Furthermore, it was pointed out that, even if IDMA process required a higher transmitter/receiver complexity, it offered sizable throughput efficiency improvement.

\section{REFERENCES}

[1] A. Sendonaris, E. Erkip, and B. Aazhang, "User cooperation diversity - part I : System description \& part II : Implementation aspects and performance analysis," IEEE Trans. Commun., vol. 51, pp. 1927-1948, Nov. 2003.

[2] A. Nosratinia, T. Hunter, and A. Hedayat, "Cooperative communication in wireless networks," IEEE Commun. Mag., vol. 42, pp. 68-73, Oct. 2004.

[3] M. Janani, A. Hedayat, T. E. Hunter, and A. Nosratinia, "Coded cooperation in wireless communications: space-time transmission and iterative decoding," IEEE Trans. Signal Processing, vol. 52, pp. 362-371, Feb. 2004.

[4] L. Ping, L. Liu, K. Y. Wu, and W. K. Leung, "Interleave Division Multiple Access (IDMA) communications," in Proc. IEEE Symp. on Turbo Codes \& Related Topics, pp. 173-180, Sept. 2003.

[5] L. Ping, L. Liu, K. Y. Wu, and W. K. Leung, "Interleave-division multipleaccess,” IEEE Trans. Wireless Commun., vol. 5, pp. 938-947, Apr. 2006.

[6] F. Babich, "Performance of hybrid ARQ schemes for the fading channel," IEEE Trans. Commun., vol. 50, pp. 1882-1885, Dec. 2002. 